with a good light which gave a view of the whole mass and the surrounding structures. A pure culture of pneumococcus was got from the pus. The apparatus, which was first described by Professor Killian* in April, 1912, and was demonstrated by him at the recent congress, has already been used considerably by Mr. Haworth $\dagger$ and Mr. E. D. Davis.f It is made in this country by Messrs. Down Bros., Limited, St. Thomas's-street, London, S.E. Queen Anne-street, W.

\section{AVOIDANCE OF SUDDEN DEATH FROM THE INDUCTION OF AN ARTIFICIAL PNEUMOTHORAX.}

By Cladde Lillingston, M.B., B.C. Cantab.

THE records of sudden death from the induction of an artificial pneumothorax are now both numerous and instructive, and the fact that the treatment has gained popularity in spite of these accidents is an index of its therapeutic value. Another sign indicative of the growing importance attached to this treatment is the space devoted to it in the advertisements and prospectuses of many leading continental sanatoriums. The treatment has also been adopted by the most important of the English sanatoriums, and I am not aware of one in which it has been practised and then abandoned, but it is to continental workers we are indebted for the most valuable observations on the various accidents which may occur.

The most serious accidents are pleural reflex, including laryngeal spasm, gas embolism, aspiration pneumonia, and an uncontrollable accidental pneumothorax due to puncture or rupture of the visceral pleura. It is not easy to estimate the incidence of these accidents, for they were more frequent in the early days of the treatment than now, and some operators have been more unfortunate than others. Forlanini in 1912 had performed 10,000 operations on 134 patients, of whom 2 died from gas embolism, and 12 exhibited serious symptoms which were very alarming in 5 cases but never fatal. Saugman in 1913, with an experience of about 5000 operations on 186 patients, had seen two fatal cases at the first injection. Vere Pearson, with an experience of 16 patients, has seen no fatal accident; and $I$, with an experience of 32 patients and several hundred punctures of the chest, have met with no more alarming symptom than slight shock of a few minutes' duration in one case. More disquieting are the reports of various French and Swedish operators whose fátalities are heavy compared with the number of patients treated. Begtrup Hansen ${ }^{1}$ has collected 8 cases of sudden death, and several isolated cases have subsequently been published.

Are these accidents inevitable, or due to faulty technique? The symptoms are numerous and varied, and consist of psychic disturbances, convulsions, ocular manifestations such as amblyopia and diplopia, mono- and hemi-plegia, rapid and irregular action of the-heart and lungs, and mottling of the skin. These conditions may disappear after a few hours to several weeks' duration, or they may rapidly terminate in death. Forlanini attributes them to pleural reflex in most cases, and to gas embolism in a few cases. Brauer, however, attaches more importance to gas embolism than to pleural reflex. It is now generally admitted that the symptoms of pleural reflex are not clinically distinguishable from those of gas embolism; and even a skilfully conducted necropsy may not be decisive, for a fatal dose of gas may have escaped from the blood-vessels of the brain before these can be examined, and, on the other hand, air may enter the blood-vessels during a necropsy, or gas may be generated in small quantities in them post mortem (Carl Sundberg). ${ }^{2}$

Gas embolism.-The bubbling sound heard when air has been drawn into a vein during an operation on the neck has never, apparently, been heard in cases of sudden death after thoracentesis; and it is also a stumbling-block to the supporters of the "embolism" school that certain mammals tolerate large intravenous injections of air. But though the importance of gas embolism may possibly have been overrated, even Forlanini ${ }^{3}$ regards it as a complication which

* Archiv für Laryngologie und Rhinologie, Band xxvi., Heft 2.

$\dagger$ Proceedings of the Royal Society of Medicine, 1913, Larpngological Section, p. 70 . may be fatal. In a case recorded by Brauer, ${ }^{4}$ a colleague attempted to induce a pneumothorax in a girl, aged 18 , by Forlanini's method. In spite of a motionless manometer, 100 c.c. of gas were forcibly injected into the chest. The patient lost consciousness, bilateral convulsions appeared, and death followed six hours after the injection. The necropsy showed small bubbles of gas uniformly distributed throughout the capillaries of the brain. The injection of gas in the absence of manometric oscillations was clearly responsible for this fatality.

In many cases of sudden death traced by the operator to embolism difficulties have been experienced in finding free pleura-the manometer has remained motionless, or there have been slight oscillations of 1 to $2 \mathrm{~cm}$. of water. The operator has now forced in gas under high pressure with a view to breaking down pleural adhesions. This procedure has occasionally been successful, and I have once adopted it at the urgent request of a patient whose disease was advanced and active, and who was prepared to take the risks involved. Ultimately a large pneumothorax was induced which rapidly reduced the fever and expectoration, and the patient, who is now under the care of Dr. Leonard Colebrook, is two years later doing well. But in view of recent fatalities due to this procedure I have not repeated it of late; and even with a large pneumothorax and characteristic manometric oscillations I do not inject gas under a pressure exceeding $20 \mathrm{~cm}$. of water. A somewhat higher pressure may be used in a partial pneumothorax. With a high pressure it is conceivable that, even if gas embolism does not occur, a pre-existing thrombus in a tuberculous area of the lung may become squeezed out and give rise to embolism, and this may be the cause of the hemiplegia which has occasionally been observed a few hours after an injection.

Brauer maintains that gas embolism may occur without the introduction of gas through the needle, provided it has wounded a blood-vessel in the lung. It certainly is conceivable that a negative pressure may exist in a pulmonary vessel surrounded by tuberculous tissue underlying pleural adhesions (see Dr. W. Parry Morgan on the physics of a tuberculous lung, THE LANĊET, July 5th, 1913), and if this be so, air under atmospheric pressure might enter from the bronch were the vessel wounded. But were a pulmonary vessel capable of aspirating air through a lesion of its walls, gas embolism should follow hæmoptysis, but there is only one such accident recorded. ${ }^{5}$ With careful technique there is no risk of gas embolism in the absence of pleural adhesions, the risk is negligible in cases of partial adhesion of the pleura, and it is considerable if the operator chooses to break down universal pleural adhesions in the absence of manometric oscillations.

Pleural reflex. - The following case of fatal pleural reflex is recorded by Professor Carl Sundberg ${ }^{b}$ :-

A woman, aged 28, suffered from pulmonary tuberculosis of the right lung for which an artificial pneumothorax was preseribed. The puncture method was employed, and seven in jections of air, amounting altogether to 2550 c.c., were given; but the air did not, apparently, (he intrapleural space. At the eighth in jection the manometer showed a negative pressure of $2 \mathrm{~cm}$. of water, but no oscillations. Before any gas had been injected the patient suddenly fainted, cunvulsions appeared, and death occurred 36 hours later. The necropsy showed no wound of the lung at the site of the injection, and no air in the heart or blood vessels. The pia mater, the cortex, the central ganglia, and the medulla were markedly hyperæmic. In the cortex of both cerebral hemispheres were numerous foci of softening, both anæmic and hæmorrhagic. The microscopic examination showed recent hyaline and granular thrombosis and commencing leucocytic emigration.

The author attributes this condition to reflex spasm of the cerebral arteries, inducing ischæmia followed by thrombosis. He has also recorded the following case :-

A man, aged 49, died three hours after the twenty-second injection of gas into a large pneumothorax. The initial pressure in the pneumothorax varied between +3 and $+7 \mathrm{~cm}$. of water, and rose to $+50 \mathrm{~cm}$. of water after 300 c.c. had been injected. In 2 to 3 minutes severe dyspncea, pallor, and a cold sweat broke out, and there was overaction of the accessory muscles of respiration. The inspiratory dyspnoea was unaccompanied by nervous phenomena or loss of consciousness. $400 \mathrm{c.c}$ of gas were withdrawn, the pressure falling from +20 to -3 . This caused only temporary improvement, The necropsy showed that the surface of the collapsed lung could not even have been touched by the uneumothorax needle owing to the size of the pneumothorax, the distance between the puncture in the parietal pleura and the lung the distance between the puncture in the parietal pleura and the lung
being $8 \mathrm{~cm}$. The pons and medulla showed marked hyperæmia and deing $8 \mathrm{~cm}$. The pons and medulla showed marked hyperæmia and degeneration of ganglion cells; but the condition to which Sundberg attaches the most importance was the post-mortem closure of the rima
glottidis, and he considers it probable that spasm of the adductor glottidis, and he considers it probable that spasm of the adductor laryng

Were these two deaths inevitable? In the second case a pressure was created within the thorax far higher than is 
now generally used; and the case is somewhat like that observed by a friend of mine who, by a mistake in the reading of the manometer, doubled the desired intrapleural pressure. His patient was unconscious for several days, but ultimately recovered. There is no reference to the use of anæsthetics in Sundberg's detailed account of his two cases. It is therefore possible that these deaths might have been averted by the use of local anæsthetics, the importance of which is illustrated by the following case quoted by Forlanini : ${ }^{7}$

The patient suffered from pleurisy with effusion. Gas was injected into the hydrothorax, and was plainly heard bubbling through the fluid. After 250 c.c. had been injected convulsions appeared. They recurred two days later when the operation was repeated. Before the next four injections the pleura was anæsthetised, and no convulsions were observed. At the seventh injection the anæsthetic was dispensed with, and alarming symptoms followed.

In this connexion it should be remarked that the bubbling of gas into a hydrothorax is unadvisable, unless as much fluid is withdrawn as gas is injected. Otherwise, the intrapleural pressure may be raised to a dangerous degree, and the point of the needle being in the fluid the manometer does not oscillate or indicate the pressure within the thorax. When a pleural effusion exists it is sometimes advisable to use two needles simultaneously, one in a dependent part of the thorax to drain the fluid, and one at a higher level to inject gas and gange the intrapleural pressure.

Still more instructive are Forlanini's experiments on 16 rabbits into whose pleuræ irritants were injected. Convulsions appeared in seven, three of which died. None of the 12 rabbits whose pleuræ were anæsthetised previous to the injection of irritants developed convulsions. In addition, therefore, to the avoidance of high intrapleural pressure, it is advisable to employ local anæsthesia at every first injection, and subsequently at every injection if the patient show signs of abnormal reflex excitability. Before every "refill" I now give omnopon by the mouth ; the immediate and after-effects of this drug are preferable to those of pure morphia.

The following "don'ts" may be briefly mentioned: 1. Don't inject gas without satisfactory manometric oscillations, or at a pressure exceeding the atmospheric pressure when beginning a first injection. 2. Don't spare anæsthetics. 3. Don't create a high intrapleural pressure. A pressure of $40 \mathrm{~cm}$. of water may cause no discomfort during an injection, but it may be more than doubled by a subsequent fit of coughing, and a leak may thus be sprung in the pneumothorax. 4. Don't induce a pneumothorax during menstruation when reflex excitability may be raised. 5. Don't puncture on the first occasion in many different places in a search for free pleura; patients have collapsed after the sixth or seventh puncture who have tolerated the first punctures well. It is better to continue the search for free pleura in a day or two. 6. Don't inject gas rapidly or at a low temperature. Brauer ${ }^{8}$ has once seen symptoms of pleural reflex after the use of cold gas. 7 . Don't use large needles or needles with rough surfaces; and don't let the rubber tubing drag on the needle during an injection. 8. Don't inject till the patient's posture is easy and comfortable. 9. Don't hesitate to withdraw the needle at the earliest sign of collapse. 10. Don't operate without brandy and a hypodermic syringe full of ether handy.

Aspiration pneumonia and accidental pneumothorax are rare. The former loomed large in medical literature more than a decade ago, when it was regarded as a serious danger to the uncompressed lung. Begtrup Hansen has collected 5 cases, including one which he has personally observed. In this case recovery was effected after some of the injected gas had been withdrawn. This complication may be a sequel to the rapid induction of a large pneumothorax at the first injection, and it is therefore sometimes associated with hæmoptysis, the arrest of which has been effected by the complete collapse of one lung by a single injection. When a pneumothorax is induced gradually the risk of aspiration pneumonia is negligible, and when violent hæmoptysis requires the immediate collapse of the whole lung the risk of aspiration pneumonia must be taken, and it is slight considering the many cases in which hæmoptysis has thus been successfully arrested.

An accidental pneumothorax may follow the introduction of the pneumothorax needle into a tuberculous cavity. Hence the advisability of introducing the needle at a point where the lung is healthy. Fortunately, when cavities lie near the surface the adjacent pleuræ are usually adherent, and when the pleuræ are non-adherent the visceral pleura and underlying lung are usually so soft and elastic that they become indented without being punctured. When a puncture of the visceral pleura occurs it seldom transmits gas. I must have pricked the lung often, but have not yet seen an accidental pneumothorax follow. This accident may also occur as the result of a too high or a too low intrapleural pressure. When the pneumothorax is not often enough refilled, the intrathoracic pressure falls considerably below atmospheric pressure, and a state of unstable equilibrium is set up. If now the patient takes violent exercise involving great inspiratory efforts, the intrathoracic pressure becomes yet more negative, and air may break through a weak spot in the visceral pleura from the bronchi and into the pleural cavity. If, on the other hand, high intrapleural pressure has been created with a view to breaking down adhesions, a portion of the visceral pleura and lung may be torn away and remain adherent to the parietal pleura. Two such cases, one of which terminated fatally in a few days, have lately been recorded. ${ }^{9}$ Forlanini has collected a few cases. The condition may simulate the escape of gas from the pneumothorax into the mediastinum, when relief can be obtained by aspirating gas from the pneumothorax. But when an accidental pneumothorax has occurred aspiration is futile if the escape of air into the pneumothorax continues.

Conclusion. - Most cases of sudden death from the induction of a pneumothorax are due to errors of technique. With care these accidents should be extremely rare, and they should not prevent the adoption of the treatment in suitable cases.

Bibliography.-1. T. Begtrup Hansen: Thesis for the Degree of M.D., Thaning \& Appel, Copenbagen. 2. C. Sundberg : Nordisk Tidsskrift for Terapi, December, 1912. 3. Forlanini : Deutsche Medizinische Wochenschrift, Dee. 14th, 1911. 4. Brauer and Spengler: Beitrage zur Klinik der Tuberkulose, Band 14, H. 4. 5. Dunin : Quoted by Begtrup Hansen. 6. O. Sundberg: Loc. cit. 7. Forlanini : Loc.
cit. 8. Brauer : Universitäts Programm, Marburg. 9. Colebrook, cit. 8. Brauer : Universitäts Programm, Marburg. 9. Colebrook,
Lillingston, Vere Pearson, and Snowdon, THE LANCET, July 15th, 1911 . Lillingston, THE LANCET, Dec. 14th, 1912.

Gorleston-on-Sea.

\section{DEGENERATION OF THE NUCLEUS LENTIFORMIS, ASSOCIATED WITH CIRRHOSIS OF THE LIVER.}

\section{BY ARTHUR T. HENRICI,}

PATHOLOGIST TO THE ST. FRANCIS HOSPITAT; DEMONSTRATOR IN PATHOLOGY, UNIVERSTTY OF PITISBURG, PITTSBURG, Pa.

UNDER the title of "Progressive Lenticular Degeneration," S. A. K. Wilson has recently described a series of cases presenting a peculiar symptomatology and revealing at necropsy characteristic lesions. For a detailed description of the disease the reader is referred to his papers, ${ }^{1}$ but the important features are described below.

The disease is "familial in the sense that it frequently attacks more than one member of a family, but is neither congenital nor hereditary." It always occurs in young people and is invariably progressive and fatal. The symptoms form a characteristic syndrome. They are : (1) bilateral involuntary movements of the extremities, sometimes also of the head and trunk, of the nature of a rhythmical tremor, increasing with volitional movement; (2) pronounced spasticity, or rather hypertonicity of the musculature, generally the face being set in a spastic smile, while the limbs are in contracture; $(3)$ in the later stages of the disease true contractures set in ; (4) dysphagia and dysarthria due to rigidity of the corresponding muscles; (5) a degree of emotionalism, the patient responding abnormally to slight stimuli ; $(6)$ there is little or no true paresis or paralysis, the patient being able to perform ordinary movements at all joints, although slowly or feebly - the reflexes are intact; and (7) transitory mental symptoms are present in some cases. The symptoms are, therefore, of a motor nature, and are extrapyramidal in origin. This symptom-complex, Wilson believes, constitutes the syndrome of the corpus striatum.

The morbid anatomy is no less characteristic than the symptomatology. There is a bilateral symmetrical softening of the nucleus lentiformis associated with oirrhosis of the 